\title{
ACTIVIDAD IN VITRO DE LA PROSTAGLANDINA F, ALFA EN EL MIOMETRIO DE ALPACAS (Lama pacos) ADULTAS NO GESTANTES
}

\author{
Gustavo Pajuelo A. ${ }^{1}$, Milder Ayón S. ${ }^{2}$ y Sergio Cueva M. ${ }^{2}$
}

\section{Ahstret}

The effect of the Prostaglandin F2 $\alpha$ (PGF2 $\alpha$ ) upon the myometrium of cranial and caudal segments from the right (RH) and left (LH) uterine horns, as well as on the right (RUB) and left (LUB) segments of the uterine body in vitro, was studied in non-pregnant adult alpacas. The results are expressed as percent of maximum tension obtained by depolarization with $50 \mathrm{mM}$ of CIK. Accumulative doses of $1.89 \times 10^{-7} \mathrm{M}, 5.66 \times 10^{-7} \mathrm{M}, 9.41$ $\times 10^{-7} \mathrm{M}$ and $1.31 \times 10^{-7} \mathrm{M}$ of the last concentration of Prostaglandin F2a, produced muscular myometrium contraction in all segments as follows: in RH 68.63 $19.32,65.42 \pm 11.73$, in LH 56.12 $18.46,47.72 \pm 6.25$, RUB $39.72 \pm 6.67$ and LUB $46.97 \pm 4.29$ for the first dose; in RH $76.18 \pm 2.91,75.97 \pm 13.04$, in LH 70.30 $\pm 7.47,66.48 \pm 6.02$, RUB 48.58 \pm 7.56 and LUB 57.27 \pm 6.49 for the second dose; in RH 83.53 $\pm 5.51,85.15 \pm 9.32$, in LH 79.35 $11.35,73.17 \pm 5.94$, RUB $45.20 \pm 5.92$ and LUB $62.50 \pm 7.20$ for the third dose; and finally, in RH 78.12 $\pm 7.25,84.53 \pm 14.68$, in LH 70.35 $\pm 11.76,61.78+3.70$, RUB $40.32 \pm 6.34$ and LUB $59.33 \pm 12.08$ for the fourth dose, respectively. In conclusion, contraction frequency (\%) was greater in the cranial and caudal segments of the right and left uterine horns $(P<0.05)$ than in the uterine body segments. Maximum contraction levels were achieved with the third accumulative dose of Prostaglandin F2 $\alpha$, but these did not exceed the contraction frequency ( $\%$ ) obtained with ClK.

Key words: Prostaglandin F2 $\alpha$, in vitro, uterine horn, muscle contraction, myometrium, non-pregnant adult alpaca.

\section{Resimen}

El presente estudio fue realizado para determinar el efecto de la Prostaglandina $\mathrm{F}_{2} \alpha$ $(\mathrm{PGF}, \alpha)$ sobre el miometrio de los segmentos craneal y caudal de los cuernos uterinos derecho (CD) e izquierdo (CI) y sobre el cuerpo uterino, segmentos derecho (CUD) e izquierdo (CUI) de alpacas adultas no gestantes en condiciones in vitro. Los resultados han sido expresados en porcentaje (\%) de máxima tensión lograda por despolarización con $50 \mathrm{mM}$ de Cloruro de Potasio. Dosis acumulativas de $1.89 \times 10^{-7} \mathrm{M}, 5.66 \times 10^{-7} \mathrm{M}, 9.41$ $\times 10^{-7} \mathrm{M}$ y $1.31 \times 10^{-7} \mathrm{M}$ de concentración final de Prostaglandina $\mathrm{F}_{2} \alpha$ produjeron contracción muscular del miometrio en todos los segmentos de la siguiente forma: Para la primera dosis en CD $68.63 \pm 9.32,65.42 \pm 11.73$, en CI $56.12 \pm 8.46,47.72 \pm 6.25$ y en CUD $39.72 \pm 6.67 \mathrm{y}$ en CUI 46.97 \pm 4.29 ; para la segunda dosis en CD 76.18 $\pm 2.91,75.97 \pm 13.04$, en CI 70.30 7.47 , 66.48 \pm 6.02 y en CUD $48.58 \pm 7.56$ en CUI $57.27 \pm 6.49$; para la tercera dosis en CD $83.53 \pm 5.51$, 85.15 \pm 9.32 , en CI 79.35 $\pm 11.35,73.17 \pm 5.94$ y en CUD $45.20 \pm 5.92$ y CUI $62.50 \pm 7.20$; y, para la cuarta dosis en CD 78.12 $\pm 7.25,84.53 \pm 14.68$, en CI $70.35 \pm 11.76,61.78 \pm 3.70$ y en CUD $40.32 \pm 6.34$ y CUI $59.33 \pm 12.08$, respectivamente. En conclusión, los segmentos craneal y

\footnotetext{
${ }^{1}$ Práctica privada

${ }^{2}$ Laboratorio de Fisiologia - FMV - UNMSM. E.mail: d170006@unmsmedu.pe
} 
caudal de los cuernos uterinos se contrajeron en mayor porcentaje $(\mathbf{P}<0.05)$ que los segmentos derecho e izquierdo del cuerpo uterino y la máxima respuesta contráctil del miometrio se observó a la tercera dosis acumulativa de Prostaglandina $\mathrm{F}_{2} \alpha$, sin sobrepasar el porcentaje de contracción lograda por $\mathrm{ClK}$

Palabras clave: Prostaglandina $F_{2} \alpha$, in vitro, cuernos uterinos, contracción muscular, miometrio, alpaca adulta no gestante.

\section{Throutuectis:}

En camélidos independientemente del lugar de ovulación, la implantación y decidualización del embrión es en el cuerno uterino izquierdo. Este hecho implica que los embriones procedentes del ovario derecho deben migrar para implantarse en el lado opuesto. Se conoce en otras especies que la Prostaglandina $\mathrm{F}_{2} \alpha$ ejerce actividad a nivel del útero, mediante la estimulación de la contracción del músculo liso (Naderali et al., 1997; Phillippe et al., 1997; Parkington et al., 1999). En camélidos, considerando la implantación preferencial de embriones en el cuerno izquierdo, es de interés conocer el efecto de la Prostaglandina $\mathrm{F}_{2} \alpha$ sobre los diferentes segmentos del cuerpo y cuernos uterinos derecho e izquierdo de alpacas adultas no gestantes.

\section{Matemies y Mtrib:}

El estudio se llevó a cabo en el Laboratorio de Anatomía y Fisiología Animal del Departamento Académico de Ciencias Veterinarias Básicas de la Facultad de Medicina Veterinaria de la Universidad Nacional Mayor de San Marcos. Se usaron seis alpacas (Lama pacos) hembras no gestantes, con un promedio de edad de cuatro años, con un parto previo, nacidas y criadas alrededor de los $4000 \mathrm{msnm}$, que fueron trasladadas a nivel del mar (200 $\mathrm{msnm})$ en donde permanecieron por un período de cuatro meses.

Las muestras para el estudio fueron obtenidas de animales previamente anestesiados. Luego de abrir la cavidad abdominal, se procedió a la medición in situ del cuerpo y cuernos uterinos. Después de separar quirúrgicamente el aparato reproductor femenino, éste fue colocado en un recipiente que contenía líquido nutricio a temperatura controlada, en donde los cuernos uterinos fueron divididos arbitrariamente en: segmentos craneal y segmentos caudal; $y$, el cuerpo uterino en: segmento lado derecho y segmento lado izquierdo. Se tomaron muestras de cada segmento, de $1 \mathrm{~cm}$ de longitud, las que fueron llevadas a un sistema de cultivo in vitro y suspendidas en $13 \mathrm{ml}$ de líquido nutricio solución Krebs-Hanseleit, mantenidos a $37^{\circ} \mathrm{C}$ con un $\mathrm{pH}$ de 7.4, aireado con una mezcla de gases de $95 \%$ de $\mathrm{O}_{2}$ y $5 \%$ de $\mathrm{CO}_{2}$. Los segmentos uterinos fueron colocados entre un tensor micrométrico y un transductor de tensión Grass tipo FT 10 y este último conectado a un registrador térmico Hewlett Packard modelo 8805 B. La tensión ejercida sobre el tejido fue de $1 \mathrm{~g}$, correspondiente a $10 \mathrm{~mm}$ del papel de registro.

Antes de la aplicación de la Prostaglandina $F_{2} \alpha$, los segmentos de miometrio acondicionados en el equipo de cultivo in vitro tuvieron un periodo de equilibración de aproximadamente $2 \mathrm{~h}$. En este periodo, el tejido fue sometido a lavados con solución KrebsHanseleit cada 10 minutos, obteniéndose la tensión óptima de contracción de $1.5 \mathrm{~g}$. La tensión óptima sirvió como base para expresar los cambios observados como respuesta del tejido a la hormona. Luego de este periodo, cada segmento uterino fue sometido a dosis aditivas de diferentes concentraciones de cloruro de potasio $(\mathrm{ClK})$ con el fin de conseguir la máxima respuesta contráctil del miometrio, la que se obtuvo a la concentración de $50 \mathrm{mM}$ de CIK. Esta máxima tensión se consideró como la máxima respuesta del tejido, $100 \%$ de la actividad de 
miometrio por despolarización de la membrana debido al potasio, y sirvió como punto referencial para obtener los porcentajes de respuesta del tejido uterino a las diferentes dosis acumulativas de la Prostaglandina $\mathrm{F}_{2} \alpha$. Luego, el tejido fue lavado 3 a 4 veces con el líquido nutricio hasta alcanzar nuevamente su nivel normal de tensión óptima. Posteriormente, los segmentos uterinos fueron sometidos a dosis acumulativas de Prostaglandina $\mathrm{F}_{2} \alpha$, administrada por medio de una micropipeta graduable, en moles, cuyas dosis fueron: $1^{\text {a }}$ dosis: $1.89 \times 10^{-7} \mathrm{M}, 2^{\mathrm{a}}$ dosis: $5.66 \times 10^{-7} \mathrm{M}, 3^{\mathrm{a}}$ dosis: $9.41 \times 10^{-7} \mathrm{M}, \mathrm{y}$; $4^{\mathrm{a}}$ dosis: $1.31 \times 10^{-6} \mathrm{M}$, de concentración final. Después de la aplicación de cada dosis de Prostaglandina $\mathrm{F}_{2} \alpha$ se dio un tiempo de respuesta de 5 minutos para luego proceder con la siguiente aplicación. La relación dosis - respuesta a la hormona por parte de los segmentos uterinos se determinó a partir de la tensión óptima. Así se registró la variación como respuesta a la actividad de la hormona.

Con el fin de comparar las respuestas de los segmentos craneal y caudal de los cuernos uterinos derecho e izquierdo y, los segmentos lado derecho y segmento lado izquierdo del cuerpo uterino se uso el análisis de varianza (ANOVA). Al resultar significativa la prueba de Anova, las diferencias específicas entre segmentos fueron analizadas mediante la prueba de Duncan $(\mathrm{P}<0.05)$.

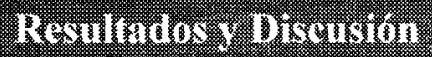

En los Cuadros 1, 2, 3 y 4 se muestran los resultados de contracción muscular lisa en los cuernos uterinos, derecho e izquierdo así como en el cuerpo uterino en respuesta a la acción de la Prostaglandina $F_{2} \alpha$.

El máximo nivel de contracción logrado no fue mayor a la máxima respuesta contráctil lograda por despolarización con ClK. Se conoce que la actividad estimuladora de la Prostaglandina $\mathrm{F}_{2} \alpha$ sobre el músculo liso se halla asociada con alteraciones en el desplazamiento del ion Cal- cio, que es inducido por despolarización de la membrana celular, lo que provoca la contracción muscular (Perusquia y Kubli-Garfias, 1992; Naderali et al., 1997; Phillippe et al., 1997; Hirata et al., 1999; Lim et al., 1999; Parkington et al., 1999). El miometrio uterino responde en diversos grados a la acción de la Prostaglandina $\mathrm{F}_{2} \alpha$, dependiendo de la especie animal, la edad y otros estados fisiológicos (Gordan et al., 1997; Navarro, 1998; Yousufzai et al., 1996; Parkington et al., 1999). La actividad contráctil de la Prostaglandina $F_{2} \alpha$ en alpacas adultas no gestantes del presente estudio fue menor en comparación a lo hallado en cobayas preñadas (Coleman y Parkington, 1988), y en cobayos prepúberes (Navarro, 1998). Esta diferencia probablemente se debe a particularidades atribuibles a la especie o al estado reproductivo de los animales estudiados.

La máxima respuesta contráctil se logró con la tercera dosis $\left(9.41 \times 10^{-7} \mathrm{M}\right)$, después de la cual la respuesta disminuyó. Esta máxima respuesta contráctil podría explicarse por la existencia de un alto grado de saturación de los receptores a esta hormona, cuyo origen en este caso es exógeno. La disminución en la contracción del tejido en la cuarta dosis podría deberse a la actividad del sistema de autorregulación de receptores, fenómeno conocido como "up and down regulation" cuyo mecanismo de regulación es aún motivo de estudio (Odore et al., 1999; Hirata et al., 1999). En los valores promedio de respuesta contráctil del miometrio a las diferentes dosis de Prostaglandina $\mathrm{F}_{2} \alpha$ (Cuadros 1, 2, 3 y 4), se observó que hubo diferencias significativas $(\mathrm{P}<0.05)$ en la contracción del músculo liso uterino de los cuernos uterinos derecho e izquierdo con respecto al cuerpo uterino, en todas las dosis estudiadas. Esta respuesta puede ser explicada por el hecho de que en los segmentos de los cuernos uterinos podrían localizarse un mayor número de receptores específicos para la Prostaglandina $\mathrm{F}_{2} \alpha$ (Perusquia y KubliGarfias, 1992; Fang et al., 1997; Phillippe et al., 1997; Baguma et al., 1998; Smith et al., 1998). 
Cuadro 1. Tensión muscular, expresada en \% de la máxima contracción lograda con CIK $(100 \%)$, en los segmentos de los cuernos uterinos derecho e izquierdo y en segmentos del cuerpo uterino de alpacas adultas no gestantes por efecto de $1.89 \times 10^{-7} \mathrm{M}$ de Prostaglandina $\mathrm{F}_{2} \alpha$.

\begin{tabular}{lcc}
\hline Segmento uterino & $\begin{array}{c}\text { Derecho } \\
\bar{X} \pm \text { D.E. }\end{array}$ & $\begin{array}{c}\text { Izquierdo } \\
\mathrm{X} \pm \mathrm{D} . \mathrm{E} .\end{array}$ \\
\hline Cuerno Craneal & $68.63 \pm 9.320^{\mathrm{A}^{*}}$ & $56.12 \pm 8.46^{\mathrm{A}}$ \\
\hline Cuerno Caudal & $65.42 \pm 11.73^{\mathrm{A}}$ & $47.72 \pm 6.25^{\mathrm{A}}$ \\
\hline Cuerpo & $39.72 \pm 6.67^{\mathrm{B}}$ & $46.97 \pm 4.29^{\mathrm{B}}$
\end{tabular}

X: Promedio; D.E.: Desviación estándar.

* Los promedios en columna con letras diferentes difieren significativamente $(\mathrm{P}<0,05)$.

Cuadro 2. Tensión muscular, expresada en \% de la máxima contracción lograda con $\mathrm{ClK}(100 \%)$, en los segmentos de los cuernos uterinos derecho e izquierdo y en segmentos del cuerpo uterino de alpacas adultas no gestantes por efecto de $5.66 \times 10^{-7} \mathrm{M}$ de Prostaglandina $\mathrm{F}_{2} \alpha$.

\begin{tabular}{lcl}
\hline Segmento uterino & \multicolumn{1}{c}{ Derecho } & $\frac{\text { Izquierdo }}{\mathrm{X} \pm \text { D.E. }}$ \\
\hline Cuerno Craneal & $76.18 \pm 2.918^{\mathrm{A}^{*}}$ & $70.30 \pm 7.47^{\mathrm{A}}$ \\
\hline Cuerno Caudal & $75.97 \pm 13.04^{\mathrm{A}}$ & $66.48 \pm 6.02^{\mathrm{A}}$ \\
\hline Cuerpo & $48.58 \pm 7.656^{\mathrm{B}}$ & $57.27 \pm 6.49^{\mathrm{B}}$ \\
\hline
\end{tabular}

$\overline{\mathrm{X}}$ : Promedio; D.E.: Desviación estándar.

* Los promedios en columna con letras diferentes difieren significativamente $(\mathrm{P}<0.05)$

Cuadro 3. Tensión muscular, expresada en \% de la máxima contracción lograda con $\mathrm{ClK}(100 \%)$, en los segmentos de los cuernos uterinos derecho e izquierdo y en segmentos del cuerpo uterino de alpacas adultas no gestantes por efecto de $9.41 \times 10^{-7} \mathrm{M}$ de Prostaglandina $\mathrm{F}_{2} \alpha$.

\begin{tabular}{lcc}
\hline Segmento uterino & $\begin{array}{c}\text { Derecho } \\
\bar{X} \pm \text { D.E. }\end{array}$ & $\begin{array}{l}\text { Izquierdo } \\
\bar{X} \pm \text { D.E. }\end{array}$ \\
\hline Cuerno Craneal & $83.53 \pm 5.51^{\mathrm{A}^{*}}$ & $79.35 \pm 11.35^{\mathrm{A}}$ \\
\hline Cuerno Caudal & $85.15 \pm 9.32^{\mathrm{A}}$ & $73.17 \pm 5.94^{\mathrm{A}}$ \\
\hline Cuerpo & $45.20 \pm 5.92^{\mathrm{B}}$ & $62.50 \pm 7.20^{\mathrm{B}}$ \\
\hline
\end{tabular}

$\overline{\mathrm{X}}$ : Promedio; D.E.: Desviación estándar.

*: Los promedios en columna con letras diferentes difieren significativamente $(\mathrm{P}<0.05)$ 
Cuadro 4. Tensión muscular, expresada en \% de la máxima contracción lograda con $\mathrm{ClK}(100 \%)$, en los segmentos de los cuernos uterinos derecho e izquierdo y en segmentos del cuerpo uterino de alpacas adultas no gestantes por efecto de $1.31 \times 10^{-6} \mathrm{M}$ de Prostaglandina $\mathrm{F}_{2} \alpha$.

\begin{tabular}{lcc}
\hline Segmento uterino & Derecho & Izquierdo \\
\hline Cuerno Craneal & $78.12 \pm 7.25^{\wedge^{*}}$ & $70.35 \pm 11.76^{\mathrm{A}}$ \\
\hline Cuerno Caudal & $84.53 \pm 14.68^{\mathrm{A}}$ & $61.78 \pm 3.705^{\mathrm{A}}$ \\
\hline Cuerpo & $40.32 \pm 6.346^{\mathrm{B}}$ & $59.33 \pm 12.08^{\mathrm{B}}$ \\
\hline
\end{tabular}

X: Promedio; D.E.; Desviación estándar.

*: Los promedios en columna con letras diferentes difieren significativamente $(\mathrm{P}<0.05)$

En los animales domésticos, la migración del huevo ó embrión es un evento común durante el período de migración embrionaria. Este fenómeno parece ser modulado por contracciones peristálticas del miometrio, siendo los estrógenos, histamina, prostaglandinas y otros productos del embrión en desarrollo los que podrían estar involucrados en la actividad del miometrio (Tominaga, 1996; Kliem et al., 1998; Stojanov y O'Neill, 1999; Tamada et al., 1999). En ratones, Yang et al., (1997) y Lim et al., (1999), encontraron que la prostaglandina $\mathrm{E}_{2}$ y $F_{2} \alpha$ juegan un rol importante para la implantación embrionaria y decidualización en el útero de estos roedores. Estas investigaciones demostraron que los genes receptores para Prostaglandina $E_{2}$ y $F_{2} \alpha$ se expresan temporalmente y en forma específica durante la periimplantación en el útero de ratones. La expresión de estos genes se observó inicialmente en el músculo circular del miometrio los días 3 a 5 de preñez, sugiriendo que esta zona es el blanco primario y punto de partida de las contracciones uterinas requeridas para el transporte, espaciamiento y/o acomodamiento embrionario en el útero, eventos precedidos por diferenciación epitelial celular, proliferación de células del estroma, edema uterino e incremento de la permeabilidad vascular endometrial localizada (Yang et al., 1997; Alvarez et al., 1999). El efecto contráctil sobre el miometrio de la Prostaglandina $\mathrm{F}_{2} \alpha$ en las alpacas adultas no gestantes ha- llado en el presente estudio nos ayuda a entender el comportamiento fisiológico que podría tener esta hormona en el útero de las alpacas y que podría estar influyendo en la implantación embrionaria.

\section{Condusiones}

La musculatura lisa del cuerpo y cuernos uterinos de la alpaca adulta no gestante es sensible a la Prostaglandina $\mathrm{F}_{2} \alpha$ y dosis acumulativas de esta hormona produjeron contracción muscular en el miometrio, cuyo máximo nivel de contracción muscular no fue mayor a la máxima respuesta contráctil lograda por despolarización de la membrana por $50 \mathrm{mM}$ de ClK, y. Los segmentos craneal y caudal de los cuernos uterinos derecho e izquierdo se contrajeron en mayor porcentaje $(\mathrm{P}<0.05)$ que los segmentos del cuerpo uterino, indicativo de la presencia de actividad en estos segmentos en alpacas no gestantes.

\section{Literatira Cthos}

1. Alvarez, R.C.; P.M. Hernandez y G.L.A. Baiza.1999. Cytokines and growth factors as autocrine and paracrine modulators in the periimplantation period. Ginecol Obstet Mex. 67: 85-93. 
2. Baguma - Nibasheka, M.; R.A. Wentworth; L.R. Green; S.L. Jenkins y P.W. Nathanielsz. 1998. Differences in the in vitro sensitivity of ovine myometrium and mesometrium to oxytocin and prostaglandins E2 and F2 alpha. Biol Repro. 58(1): 73-78.

3. Coleman H.A. y H.C. Parkington. 1988. Induction of prolonged excitability in myometrium of pregnant guinea-pigs by prostaglandin F2 alpha. J Physiol (Lond) 399: 33-47.

4. Fang X.; S. Wong y B. F. Mitchell. 1997. Effects of RU486 on estrogen, progesterone, oxytocin, and their receptors in the rat uterus during late gestation. Endocrinology. 138(7): 2763-2768.

5. Fernández-Baca, S. 1993. Manipulation of reproductive funtions in male and female new world camelids. Animal Reproduction Science. 3: 307323.

6. Gordan P.L.; S.L. Jenkins; R.A. Wentworth y P.W. Nathanielsz. 1997. Effect of in vivo estradiol administration to billaterally ovariectomized rats on in vitro myometrial responsiveness to prostaglandin F2 alpha and oxytocin. Biol Repro. 57(3): 597-601.

7. Hirata, Y.; A. Kanada; H. Miyai; Y. Mabuchi y K. Aisaka. 1999. Effects of the new potassium channel opener JTV506 on coronary vessels in vitro and in vivo. Arzneimittelforschung. 49(3): 199206.

8. Kliem, A.; F. Tetens; T. Klonisch; M. Grealy y B. Fischer. 1998. Epidermal growth factor receptor and ligands in elongating bovine blastocysts. Mol Reprod Dev. 51(4): 402-412.

9. Lim, H.; R.A. Gupta; W.G Ma; B.C. Paria; D.E. Moller; J.D. Morrow; R.N. DuBois; J.M. Trzaskos y S.K. Dey. 1999. Cyclo-oxygenase-2-derived prostacyclin mediates embryo implantation in the mouse via PPAR delta. Genes Dev. 13(12): 1561-1574.

10. Naderali, E.K.; N. Buttell; M.J. Taggart; A.J. Bullock; D.A. Eisner y S. Wray. 1997. The role of sarcolemmal
$\mathrm{Ca}(2+)$ - ATPase in the $\mathrm{pH}$ transients associated with contraction in rat smooth muscle. J Physiol (Lond) 1; 505(Pt 2): 329-336.

11. Navarro, G.P. 1998. Efecto de la Prostaglandina F2a sobre la musculatura uterina del cobayo (Cavia porcellus) prepuber. Tesis Medico Veterinario. Facultad de Medicina Veterinaria Universidad Nacional Mayor de San Marcos. 35 $\mathrm{p}$.

12. Odore, R.; G Re; P. Badino; A. Donn; D. Vigo; B. Biolatti y C. Girardi. 1999. Modifications of receptor concentrations for adrenaline, steroid hormones, prostaglandin $\mathrm{F} 2$ alpha and gonadotropins in hypophysis and ovary of dairy cows with ovarian cysts. Pharmacol Res. Vol 39 (4): 297-304.

13. Parkington, H.C.; M.A. Tonta; N.K. Davies; S.P. Brennecke y H.A. Colemen. 1999. Hyperpolarization and slowing of the rate of contraction in human uterus in pregnancy by prostaglandins E2 and F2 alpha: involvement of the Na pump. J Physiol (Lond) 515(Pt 1): 229-243.

14. Perusquia, M. y C. Kubli-Garfias. 1992. External calcium dependence of the uterine contraction induced by prostaglandins E2 and F2 alpha and its antagonism with natural progestins. Prostaglandins. 43(5): 445-455.

15. Phillippe, M.; T. Saunders y A. Basa. 1997. Intracellular mechanisms underlying prostaglandin $\mathrm{F} 2$ alpha-stimulated phasic myometrial contracctions. Am J Physiol. 273(4 Pt 1): E665-E673.

16. Smith, G.C.; M. Baguma-Nibasheka; W.X. Wu y P.W. Nathanielsz. 1998. Regional variations in contractile responses to prostaglandins and prostanoid receptor messenger ribonucleic acid in pregnant baboon uterus. Am J Obstet Gynecol. 179(6 Pt 1): 1545-1552.

17. Stojanov, T. y C. O'Neill. 1999. Ontogeny of expression of a receptor for platelet - activating factor in mouse preimplantation embryos and the effects of fertilization and culture in vitro on its 
expression. Biol Reprod. 60(3): 674-682.

18. Tamada, H.; C. Higashiyama; Takano; N. Kawate; T. Inaba y T. Sawada. 1999. The effects of heparin binding epidermal growth factor like growth factor on preimplantation embryo development and implantation in the rat. Life Sci. 64(22): 1967-1973.

19. Tominaga, T. 1996. Studies on the mechanism of embryo implantation. Nippon Sanka Fujinka Gakkai Zasshi. 48(8): 591-603.
20. Yang, Z.M.; S.K. Das; J. Wang; Y. Sugimoto; A. Ichikawa y S.K. Dey. 1997. Potential sites of prostaglandin actions in the periimplantation mouse uterus: differential expression and regulation of prostaglandin receptor genes. Biol Reprod. 56(2): 368-379.

21. Yousufzai, S.Y.; Z. Ye y A.A. AbdelLatif. 1996. Prostaglandin F2 alpha and its analogs induce release of endogenous prostaglandins in iris and ciliary muscles isolated from cat and other mammalian species. Exp Eye Res. 63(3): 305-310. 